\title{
Evidence of Cryogenic Indentation-Induced Grain Growth in Highly Twinned Nanocrystalline Copper
}

\author{
J.G. Brons ${ }^{1}$, H.A. Padilla ${ }^{2}$, G.B. Thompson ${ }^{1}$, B.L. Boyce ${ }^{2}$ \\ 1. The University of Alabama, Metallurgical Engineering Department, Tuscaloosa, AL 35401, USA \\ 2. Sandia National Laboratories, Albuquerque, NM 87123, USA
}

Grain growth is a product of a driving force and boundary mobility. These driving forces can originate from stored deformation energy, grain boundary energy, surface energy, elastic energy, or chemical and/or thermal gradients [1]. The boundary mobility is representative of the kinetics necessary to allow the system to respond to the applied driving force. A lack of sufficient mobility and/or driving force can prevent a system from reaching the microstructural configuration with the lowest energy. Since the mobility is commonly related to atomic diffusion, temperatures of approximately one-half or higher of the homologous temperature are typically required for substantial grain growth. During grain growth, if the grain size distribution remains self-similar, it is referred to as normal growth. In some cases, however, a single grain or an aggregate of grains can coarsen at a rate faster than others in the microstructure and is referred to as abnormal grain growth.

As the grain size of a material approaches the nanometer regime, the microstructure can exhibit peculiar grain growth behavior. For example, nanocrystalline metals have been shown to undergo both moderate, widespread coarsening as well as dramatic abnormal grain growth as a result of tensile deformation [2], high-cycle fatigue [3], low-cycle fatigue [4], and indentation [5] at moderate to low homologous temperatures. In fine-grained $\mathrm{Cu}$, samples that are left at room temperature have been shown to display abnormal coarsening. Zhang et al. [5] previously reported transmission electron microscopy (TEM) studies where abnormal grain growth at room temperature occurred under in-situ indentation. In their report, the starting grain size was $40 \mathrm{~nm}$. After indentation, certain grains achieved sizes of nearly 300 $\mathrm{nm}$. Even more surprising was a series of ex-situ cryogenic indentations at $-190^{\circ} \mathrm{C}$ where the grains grew up to $700 \mathrm{~nm}$. At temperatures of a few hundred ${ }^{\circ} \mathrm{C}$, typical boundary velocities for $\mathrm{Cu}$ are on the order of $10^{-9} \mathrm{~m} / \mathrm{s}$ [1]; at $-190^{\circ} \mathrm{C}$ one might expect grain boundary migration to occur only on geologic timescales. To date, the ability for grains to grow under cryogenic indentation, where temperatures are too low for reasonable mobility, is not well understood.

In this research, a series of nanocrystalline $\mathrm{Cu}$ films with a high density of twin boundaries $(\Sigma 3)$ were subjected to indentation while submerged in liquid nitrogen at $77 \mathrm{~K}$. Subsequent quantification using precession-enhanced electron diffraction in the TEM determined the crystallographic texture and grainto-grain misorientation. The grain boundary misorientations were quantified in order to understand which grain boundary types are present after cryogenic mechanically-induced grain growth.

Three distinct regions were identified, as seen in Figure 1: (i) undeformed, which preserved the parent microstructure, (ii) pile-up (the area of mass build up around the sides of the indent) and (iii) under the indent. As shown in Figure 2, the grains in the undeformed film retained a high density of $\Sigma 3$ boundary twins, whereas the pile-up region showed grain coarsening, prevalent $\Sigma 7$ subgrain orientations and an order of magnitude decrease in $\Sigma 3$ twin boundaries. Coupling these experimental results with prior simulations [6], a detwinning mechanism is believed to be a contributing mechanism that allowed the 
grains to coarsen at significantly low homologous temperature, where diffusion-based mobility would be inactive.

References:

[1] Gottstein, G. and L.S. Shvindlerman, Grain boundary migration in metals: Thermodynamics, kinetics, applications 1999, Boca Raton: CRC Press.

[2] Gianola, D.S., et al., Scripta Mater, (55) 2006, 649-652.

[3] Boyce, B.L. and H.A. Padilla. Metall Mater Trans A, (42A) 2011, 1793-1804.

[4] Hoppel, H.W., et al.. Phil Mag, (82) 2002, 1781-1794.

[5] Zhang, K., et al.. Appl Phys Lett, (87) 2005, 061921.

[6] Harper, J.M.E., et al.,. J Appl Phys, (86) 1999, 2516-2525.

[7] Brons, J.G. et al., Scripta Mater in press (2013).

[10] This work has been supported by the US Department of Energy, Office of Basic Energy Sciences. JGB and GBT received additional supplementary support from NSF-EPS-0814103. Sandia is a multiprogram laboratory operated by Sandia Corporation, a Lockheed Martin Company, for the United States Department of Energy's National Nuclear Security Administration under contract DE-AC04-94AL85000.

(a)

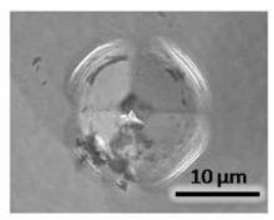

(b)

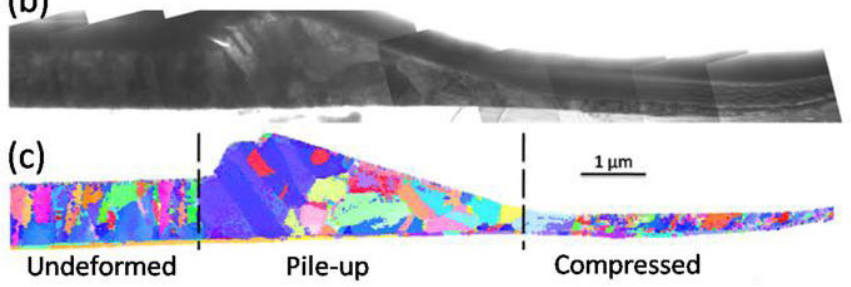

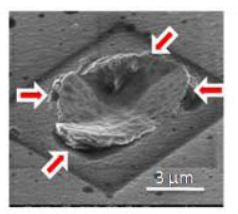
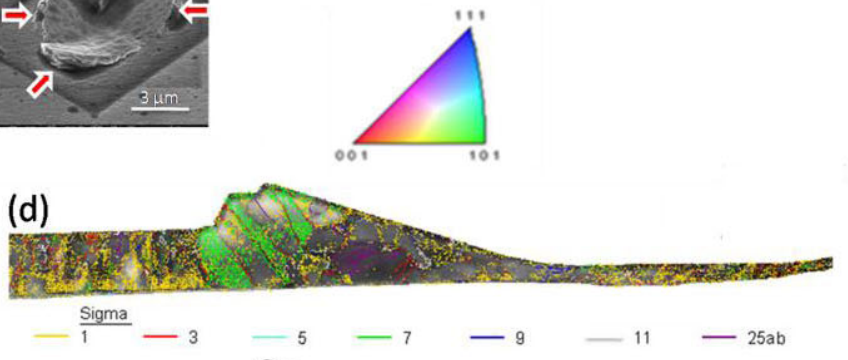

(e)

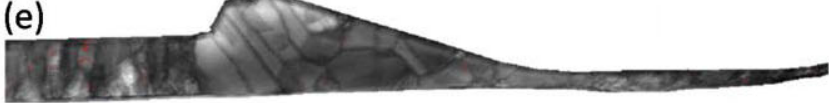

Figure 1. Indented nanotwinned $\mathrm{Cu}$ film in (a) SEM image of indent with arrows showing site specific foil lift-out locations

(b) TEM bright field image (c) inverse pole figure orientation map (d) image quality map with CSL boundaries highlighted

(e) image quality map with twin boundaries highlighted in red.

(a)

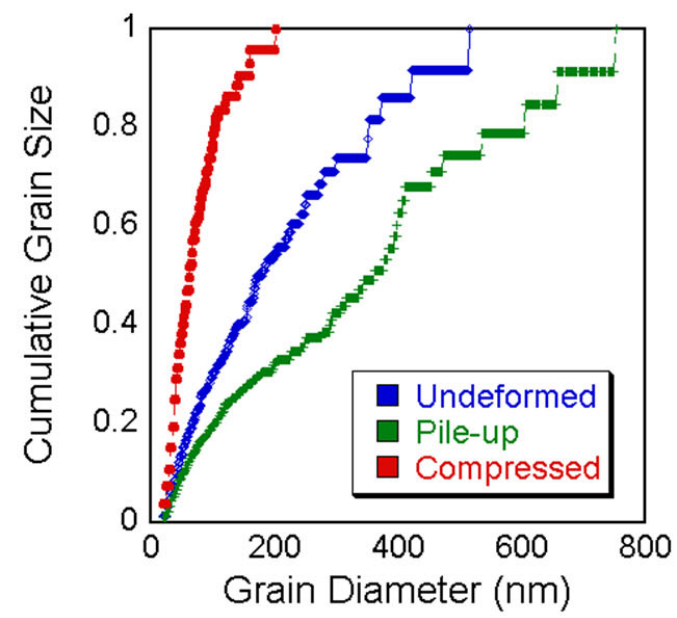

(b)

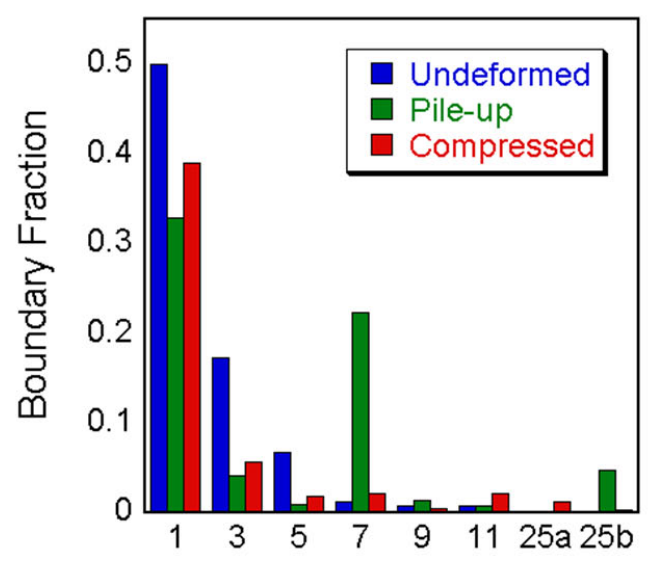

Coincidence Site Lattice $(\Sigma)$ Value

Figure 2. Region-specific response of nanotwinned $\mathrm{Cu}$ to mechanical indentation at cryogenic temperatures (a) grain size evolution and (b) CSL boundary evolution 